\title{
Representation of gradients of a scalar field on the sphere using a 2D Fourier expression
}

\author{
M. A. Sharifi ${ }^{\text {a, b }}$, K. Ghobadi-Far ${ }^{\text {a, } *}$ \\ ${ }^{a}$ School of Surveying and Geospatial Engineering, University college of engineering, University of Tehran, North Kargar Ave., P.O. \\ Box 11365-4563, Tehran, Iran - (sharifi, k.ghobadi)@ut.ac.ir \\ ${ }^{\mathrm{b}}$ Research Institue of Geoinformation Technology (RIGT), University college of engineering, University of Tehran, North Kargar \\ Ave., Tehran, Iran
}

KEY WORDS: 2D Fourier series, gradients, representation coefficients, D-functions, spectral transfers

\begin{abstract}
:
Representation of data on the sphere is conventionally done using spherical harmonics. Making use of the Fourier series of the Legendre function in the SH representation results in a 2D Fourier expression. So far the 2D Fourier series representation on the sphere has been confined to a scalar field like geopotential or relief data. We show that if one views the 2D Fourier formulation as a representation in a rotated frame, instead of the original Earth-fixed frame, one can easily generalize the representation to any gradient of the scalar field. Indeed, the gradient and the scalar field itself are simply linked in the spectral domain using spectral transfers. We provide the spectral transfers of the first-, second- and third-order gradients of a scalar field in a local frame. Using three numerical examples based on gravity and geometrical quantities, we show the applicability of the presented formulation.
\end{abstract}

\section{INTRODUCTION}

The spherical harmonics are usually employed to represent geoscience data globally on the sphere. In particular, in the case of harmonic scalar fields like gravitational potential, since the spherical harmonics are the solutions of the Laplace equation in spherical coordinates, the most appropriate choice is the representation in terms of spherical harmonic series (Heiskanen and Moritz, 1967). Due to the characteristics of the spherical harmonics such as orthogonality and global support, this representation is of great advantage in all fields of geoscience. The explicit link in the spectral domain between the gradients of a scalar field and the field itself can only be established in the case of isotropic functionals like pure radial derivatives.

An alternative formulation of a scalar field on the sphere is derived by expanding the Legendre function in the spherical harmonic representation as a series in sine and cosine terms (e.g., Schuster, 1903; Ricardi and Burrows, 1972; Colombo, 1981; Sneeuw and Bun, 1996). This alternative formulation is a 2D Fourier expression in terms of spherical longitude and latitude. The 2D Fourier methods are mostly appropriate for those applications on the sphere which the problem can not be solved explicitly using spherical harmonic series. For instance, the problem of global spherical harmonic analysis of anisotropic functionals can not be dealt with easily and explicitly using spherical harmonics. As an another example, the aliasing problem on the sphere is better solved and explained based on the Fourier series representation (Jekeli, 1996).

The point about the 2D Fourier series representation in terms of spherical coordinates is that so far in the literaure it has been confined to a scalar field like geopotential or its radial derivatives (e.g., Gruber et al., 2011).

In this paper, we provide a general Fourier formulation for representing the gradients of a scalar field on the sphere. In other words, a 2D Fourier series representation is presented that explicitly deals with any gradients of the field (Ghobadi-Far et al., 2015). In particular, we show that if the Fourier series representation is viewed as a representation in the rotated frame, instead of the original Earth-fixed frame, the generalization to any functional of the scalar field can be done easily. In order to derive the representation in the rotated frame the representation coefficients are employed (Wigner, 1959).

This paper starts with an introduction to the representation coefficients and their application to rotate the spherical harmonics (section 2). In section 3, the formulation in the rotated frame is derived. Moreover, the spectral transfers of the gradients up to the third order are given in this section. Section 4 provides three numerical examples of the 2D Fourier series. Finally, the conclusions are drawn in the last section.

\section{REPRESENTATION COEFFICIENTS}

To transform the spherical harmonics to a rotated frame one can make use of the representation coefficients (Wigner, 1959). Sneeuw (1992) emplys of the representation coefficients to derive a representation of the gravitational functionals along the orbit in terms of Kepler elements. The along-orbit representation is obtained by rotating the spherical harmonics from Earth-fixed frame to a rotating one.

In an almost similar way to (Sneeuw, 1992), we use the representation coefficients to obtain a 2D Fourier expression of a scalar field in spherical coordinates.

Suppose the original and rotated frame are transformed into each other through the Eulerian rotation sequence:

$$
R(\alpha, \beta, \gamma)=R_{3}(\gamma) R_{2}(\beta) R_{3}(\alpha)
$$

where the $R_{3}$ and $R_{2}$ matrices are the rotation matrices about the $\mathrm{z}$ - and $\mathrm{y}$ - axis, respectively. Then, the relation between the spherical harmonics in the original $(\varphi, \lambda)$ and rotated $\left(\varphi^{\prime}, \lambda^{\prime}\right)$ frame is given by (Sneeuw, 1992):

\footnotetext{
* Corresponding author
} 


$$
\bar{Y}_{n m}(\varphi, \lambda)=\sum_{k=-n}^{n} \bar{D}_{n m k}(\alpha, \beta, \gamma) \bar{Y}_{n k}\left(\varphi^{\prime}, \lambda^{\prime}\right)
$$

One can see that the spherical harmonics in the original frame are a linear combination of the same degree of those in the rotated frame. The representation coefficients $\bar{D}_{n m k}$ are defined as:

$$
\bar{D}_{n m k}(\alpha, \beta, \gamma)=e^{i m \alpha} \bar{d}_{n m k}(\beta) e^{i k \gamma}
$$

with $\bar{d}_{n m k}$ being the Wigner-d coefficients (Sneeuw, 1992). Inserting Eq. (2) in a spherical harmonic series provides the required representation in the rotated frame.

\section{FOURIER SERIES FORMULATION}

In order to obtain a 2D Fourier series representation of a scalar field the spherical harmonic series is transformed to a rotated frame. Thus, the starting point of the derivation is the series representation in spherical harmonics:

$$
V(r, \varphi, \lambda)=\frac{G M}{R} \sum_{n=0}^{N}\left(\frac{R}{r}\right)^{n+1} \sum_{m=-n}^{n} \bar{K}_{n m} \bar{Y}_{n m}(\varphi, \lambda)
$$

where $\bar{Y}_{n m}(\varphi, \lambda)=\bar{P}_{n m}(\sin \varphi) e^{i m \lambda}$ and $\bar{K}_{n m}$ are the spherical harmonic coefficients of the geopotential $V$. Please note that although the series expression of the geopotential is considered here, one can consider any other scalar field on the sphere.

The 2D Fourier series formulation is obtained by setting three Euler angles as $\alpha=\lambda-\pi / 2, \beta=\pi / 2, \gamma=\pi / 2$. Applying these rotations results in a rotated frame in which its equatorial plane coincides with the local meridian plane of the evaluation point. For more details, see (Ghobadi-Far et al., 2015). Thus, from Eq. 2 one derives the following formula for associated Legendre functions:

$$
\bar{P}_{n m}(\sin \varphi)=\sum_{k=-n}^{n} a_{n m k} e^{i k \varphi}
$$

Where $a_{n m k}$ are the Fourier coefficients of the Legendre function given by:

$$
a_{n m k}=i(k-m) \bar{d}_{n m k}(\pi / 2) \bar{P}_{n k}(0)
$$

with $\bar{P}_{n k}(0)$ being the associated Legendre function at the equator.

Finally, substituting Eq. (5) in (4) gives us the 2D Fourier series formulation

$$
\begin{gathered}
V(r, \varphi, \lambda)=\frac{G M}{R} \sum_{n=0}^{N}\left(\frac{R}{r}\right)^{n+1} \sum_{\substack{m=-n k=-n \\
\sum_{n m k}}}^{n} \bar{K}{ }^{i(k \varphi+m \lambda)} \\
a^{i(7)}
\end{gathered}
$$

The aim of this paper is to introduce a general formulation that represents any functional of the scalar field $V$ as a 2D Fourier expression. In fact, due to the special rotated frame chosen for each external point, one is able to link any functional of the scalar field to the field itself in the spectral domain using spectral transfers $h_{n k}^{f}$.

Basically, the following formula can be used to represent any functional $f$ of the scalar field as a $2 \mathrm{D}$ Fourier series.

$$
\begin{array}{r}
f(r, \varphi, \lambda)=\frac{G M}{R} \sum_{n=0}^{N}\left(\frac{R}{r}\right)^{n+1} \sum_{m=-n k=-n}^{n} \sum_{m m}^{n} \bar{K} \\
h_{n k}^{f} a_{n m k}^{(*)} e^{i(k \varphi+m \lambda)}
\end{array}
$$

where

$$
a_{n m k}^{*}=i^{(k-m)} \bar{d}_{n m k}(\pi / 2) \bar{P}_{n k}^{\prime}(0)
$$

with $\bar{P}_{n k}^{\prime}(0)$ being the first derivative of the associated Legendre function at the equator.

The spectral transfers $h_{n k}^{f}$ of the first-, second- and third-order gradients in a local frame are obtained by applying the differential operators of the functionals (e.g., Šprlák and Novák, 2014) to equation (7) based on the mechanism and procedure presented by Sneeuw (2000). The spectral transfers of the mentioned gradients are given in Table (1) (Ghobadi-Far et al., 2015). Note that the local frame used here is the Local NorthOriented reference Frame (LNOF). The LNOF is a moving frame with its origin located at evaluation point and the $\mathrm{x}-, \mathrm{y}-$ and $\mathrm{z}$-axis pointing to the north, west and radially upward, respectively.

As can be seen, only gradients that related to the field $V$ through an odd derivative with respect to the y-coordinate of the LNOF are associated with the $a_{n m k}{ }^{*}$ coefficients (see Eq. (9)). For all other gradients the $a_{n m k}$ coefficients are involved.

The collection of spectral transfers listed in Table (1) can be seen as a pocket guide that provides the spectral properties of the functionals of a scalar field. These spectral transfers can be used for spectral analyses on the sphere such as global spherical harmonic synthesis and analysis, contribution analysis, sensitivity analysis, etc. 
Table 1. The spectral transfers of the first-, second- and thirdorder gradients of a scalar field in LNOF

\begin{tabular}{|c|c|c|}
\hline gradient & $h_{n k}^{f}$ & $a_{n m k}^{(*)}$ \\
\hline$V$ & 1 & $a_{n m k}$ \\
\hline$V_{x}$ & $\frac{1}{r}[\mathrm{ik}]$ & $a_{n m k}$ \\
\hline$V_{y}$ & $\frac{1}{r}[1]$ & $a_{n m k}^{*}$ \\
\hline$V_{z}$ & $\frac{1}{r}[-(n+1)]$ & $a_{n m k}$ \\
\hline$V_{x x}$ & $\frac{1}{r^{2}}\left[-\left(k^{2}+\mathrm{n}+1\right)\right]$ & $a_{n m k}$ \\
\hline$V_{x y}$ & $\frac{1}{r^{2}}[\mathrm{ik}]$ & $a_{n m k}^{*}$ \\
\hline$V_{x z}$ & $\frac{1}{r^{2}}[-\mathrm{ik}(\mathrm{n}+2)]$ & $a_{n m k}$ \\
\hline$V_{y y}$ & $\frac{1}{r^{2}}\left[k^{2}-(\mathrm{n}+1)^{2}\right]$ & $a_{n m k}$ \\
\hline$V_{y z}$ & $\frac{1}{r^{2}}[-(\mathrm{n}+2)]$ & $a_{n m k}^{*}$ \\
\hline$V_{z z}$ & $\frac{1}{r^{2}}[(n+1)(\mathrm{n}+2)]$ & $a_{n m k}$ \\
\hline$V_{x x x}$ & $\frac{1}{r^{3}}\left[-i k\left(k^{2}+3 n+5\right)\right]$ & $a_{n m k}$ \\
\hline$V_{x x y}$ & $\frac{1}{r^{3}}\left[-\left(k^{2}+\mathrm{n}+1\right)\right]$ & $a_{n m k}^{*}$ \\
\hline$V_{x x z}$ & $\frac{1}{r^{3}}\left[k^{2}(n+3)+(\mathrm{n}+1)(\mathrm{n}+3)\right]$ & $a_{n m k}$ \\
\hline$V_{x y y}$ & $\frac{1}{r^{3}}\left[\mathrm{ik}\left(k^{2}-(\mathrm{n}+1)^{2}\right)\right]$ & $a_{n m k}$ \\
\hline$V_{x y z}$ & $\frac{1}{r^{3}}[-\mathrm{ik}(\mathrm{n}+3)]$ & $a_{n m k}^{*}$ \\
\hline$V_{x z z}$ & $\frac{1}{r^{3}}[\mathrm{ik}(\mathrm{n}+2)(\mathrm{n}+3)]$ & $a_{n m k}$ \\
\hline$V_{y y y}$ & $\frac{1}{r^{3}}\left[k^{2}-(\mathrm{n}+2)^{2}-1\right]$ & $a_{n m k}^{*}$ \\
\hline$V_{y y z}$ & $\frac{1}{r 3}\left[(\mathrm{n}+1)^{2}(n+3)-k^{2}(n+3)\right]$ & $a_{n m k}$ \\
\hline$V_{y z z}$ & $\frac{1}{r^{3}}[(n+2)(\mathrm{n}+3)]$ & $a_{n m k}^{*}$ \\
\hline$V_{z z z}$ & $\frac{1}{r^{3}}[-(\mathrm{n}+1)(n+2)(\mathrm{n}+3)]$ & $a_{n m k}$ \\
\hline
\end{tabular}

\section{NUMERICAL EXAMPLES}

Three numerical examples are provided in this section to show the applicability of the Fourier series representation. For more examples see (Ghobadi-Far et al., 2015).
The first example consists of ten gradients of the third-order tensor synthesized from the EIGEN-6C geopotential model (Shako et al., 2014) to degree and order 360. Results are shown in Figure (1). These maps show the spatial representation of each individual component of the third-order tensor. One can see the strength of some gradients compared to others. The third-order gradients can be used for upward/downward continuation of geodetic and geophysical data. For instance, one can employ the third-order gradients to do the analytical continuations of the second-order gradients in both radial and lateral directions.

Finally, it is worth mentioning that comparing the spherical harmonic representation of the third-order gradients (Šprlák and Novák, 2014) to the Fourier series formulation reveals the compactness, efficiency and much less complexity of the gradients in the latter formulation. This is because in the Fourier series representation each gradient is linked to the scalar field using a spectral transfer.

The second example deals with the global spherical harmonic analysis of the Digital Elevation Models (DEM). For this purpose, we use ETOPTO1 global relief model which consists of 1 arcmin land topography and ocean bathymetry (Amante and Barry, 2009) (see Figure (2)). The ETOPTO1 model is resampled to a grid with cell size of about 5 arcmin. Then, the global spherical harmonic analysis is carried out to determine the spherical harmonic coefficients of the relief data.

The coefficients are estimated to degree and order 2000. The degree amplitude and individual coefficients are shown in the figure. Moreover, the difference between the original ETOPO1 and the synthesized data are also shown. The RMS of the differences is $52 \mathrm{~m}$ globally. As can be seen, the borders between continents and oceans and also regions with high anomalies are highlighted in the differences. The slow decay of the signal power in the spectral domain is also visible in the results.

The last example is concerned with the sensitivity analysis of some regularly-used functionals in physical geodesy. One can evaluate the sensitivity of a functional to a certain coefficient using the sensitivity analysis. See (Sneeuw, 2000) for more details. It should be emphasized that the sensitivity analysis is a forward modelling tool. In other words, it maps harmonic coefficients to the functionals. It does not provide any information about the recoverability of the potential coefficients (Sneeuw, 2000).

As an example, a sensitivity analysis is employed for geoid undulations, gravity anomaly and deflections of the vertical based on the EIGEN-6C model to degree and order 500. The results are shown in Figure (3). The spectral transfers of the four functionals are obtained from those of the geopotential and its first derivatives.

As expected, the maximum signal size of the geoid undulations and gravity anomaly corresponding to the harmonic coefficients decays as the degree increases. The contribution of coefficients lower than 20 is the most for these two functionals. Moreover, the contribution of the harmonic coefficients varies homogeneously with degree $n$, as it is expected due to the isotropic spectral transfers of these two functionals.

However, in the case of vertical deflections the situation is different. The north-south vertical deflection is mostly sensitive to the zonal and near zonal coefficients. While, in the case of eastwest vertical deflection, the maximum signal size attributed to the sectorial and near sectorial coefficients is high and that of the near zonal coefficients is low. The results show that in order to model N-S vertical deflection, it is advantageous to use a geopotential model that the accuracy of its zonal and near zonal coefficients is better than others. Contrarily, a model with more accurate sectorial and near sectorial coefficients is superior if one aims to model E-W vertical deflection. 


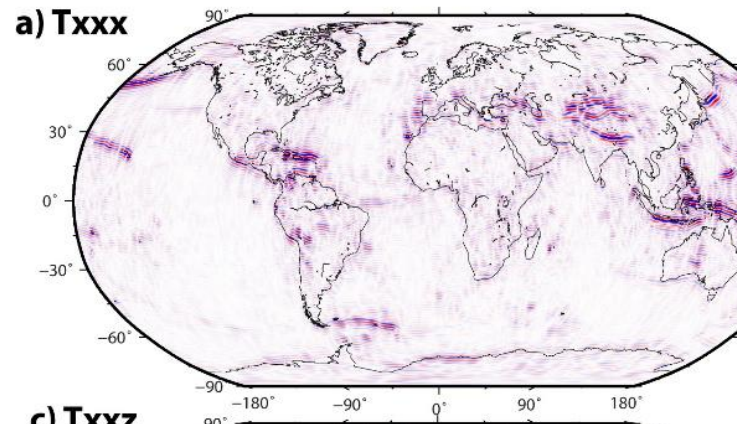

b) Txxy
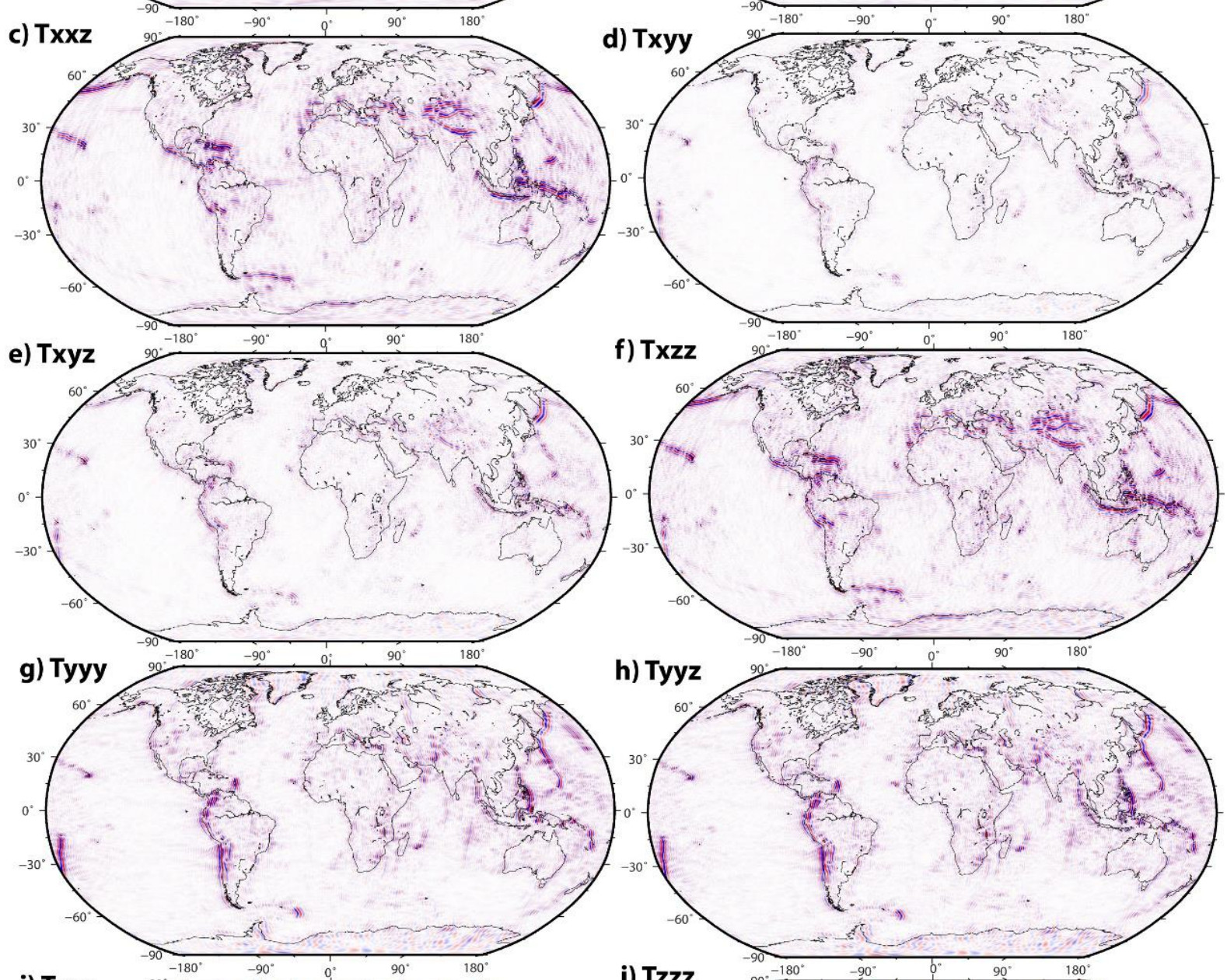

h) Tyyz
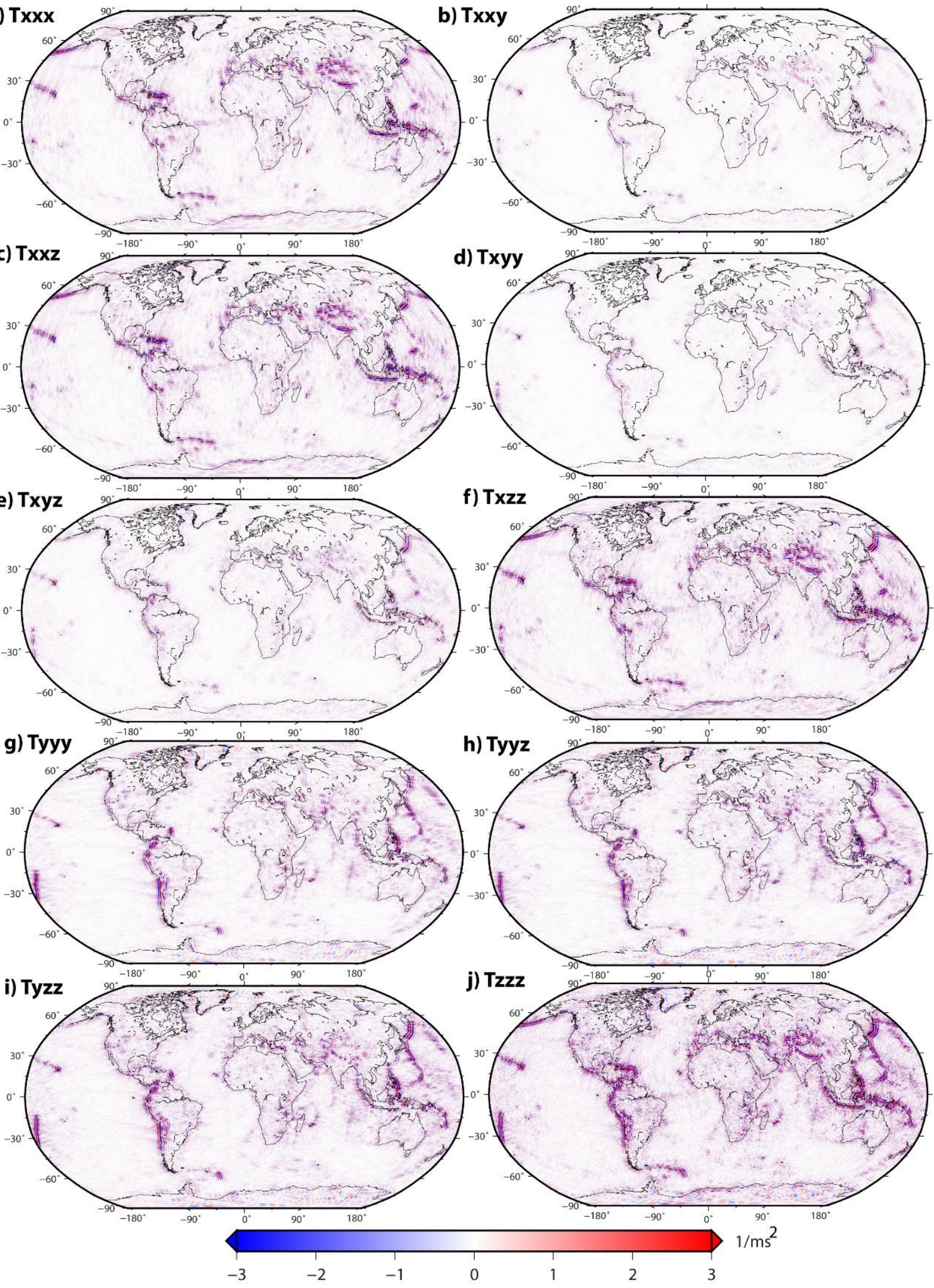

j) Tzzz

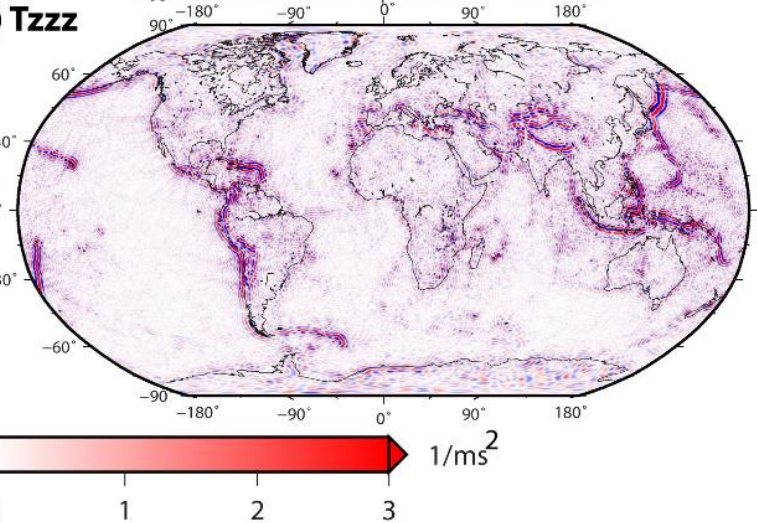

Figure 1. Maps of the third-order gradients synthesized from the EIGEN-6C model to degree and order 360. The GRS80 model is used as the normal field 
a) ETOPO1

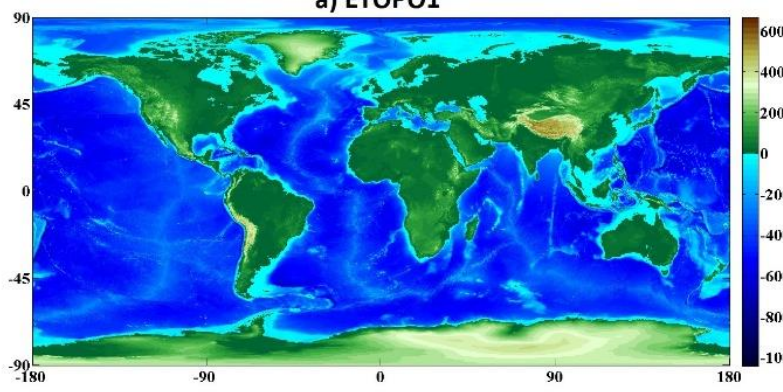

c) degree amplitude

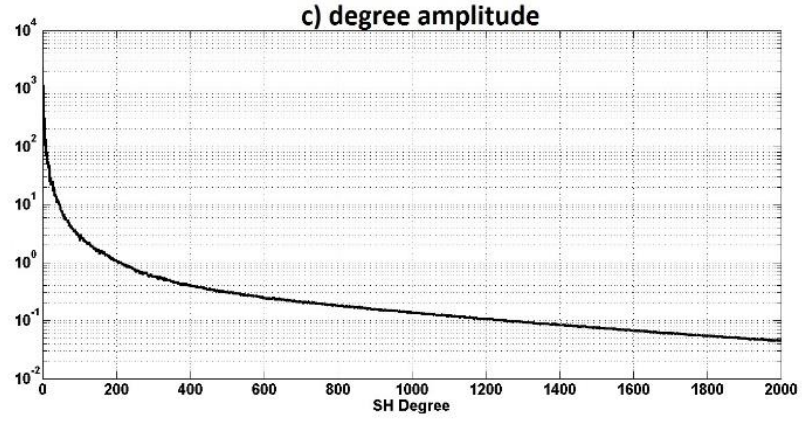

b) difference

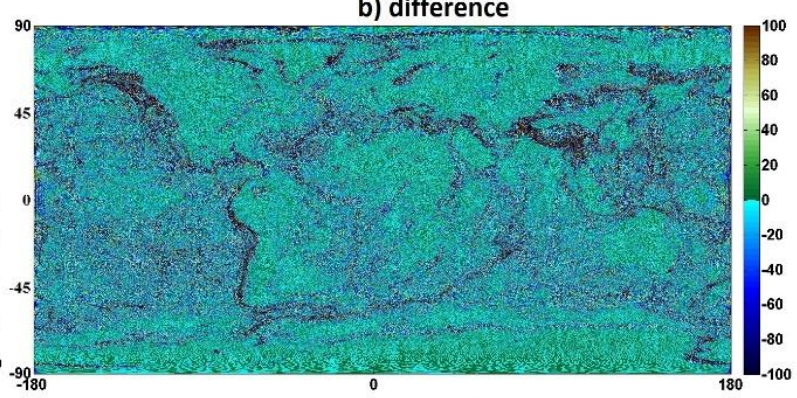

d) individual coefficients

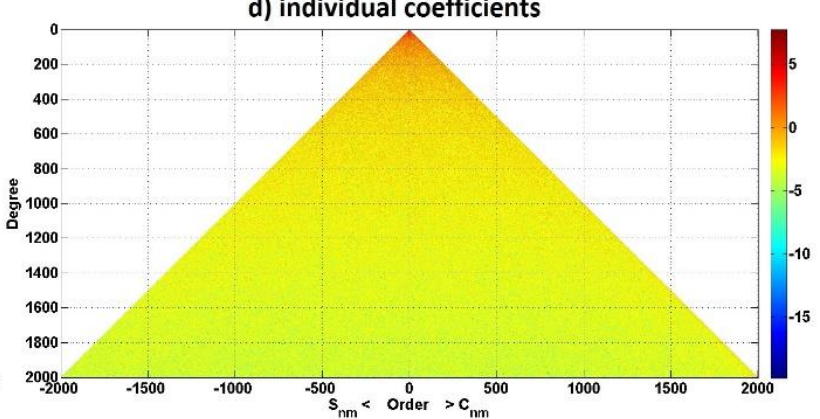

Figure 2. a) ETOPO1 global relief model in $\mathrm{m}$, b) difference between the original ETOPO1 and synthesized model from estimated coefficients in $\mathrm{m}, \mathrm{c}$ ) degree amplitude of the estimated coefficients and d) individual coefficients in $\log _{10}$ scale

a) geoid undulations

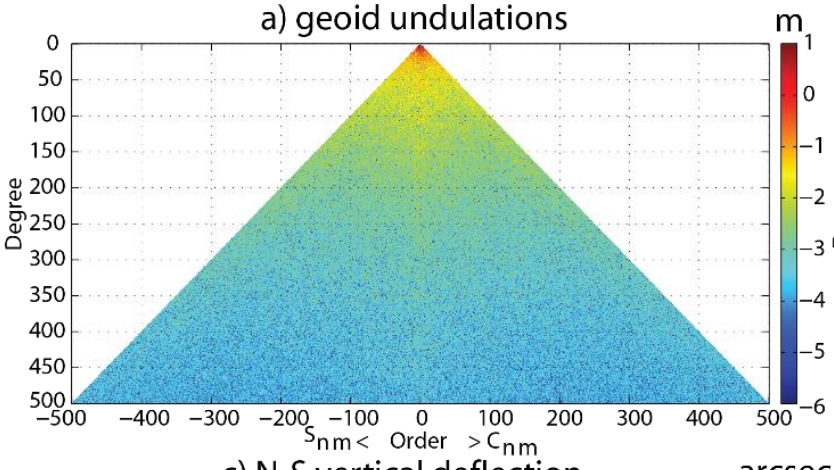

c) N-S vertical deflection

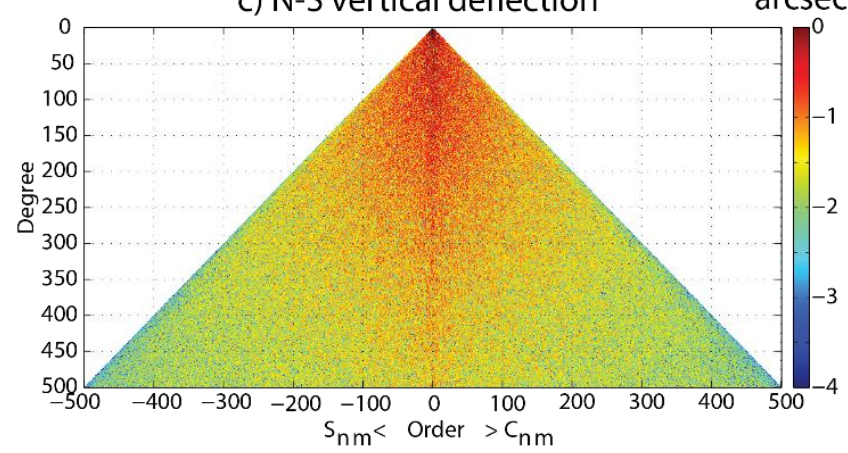

b) gravity anomaly

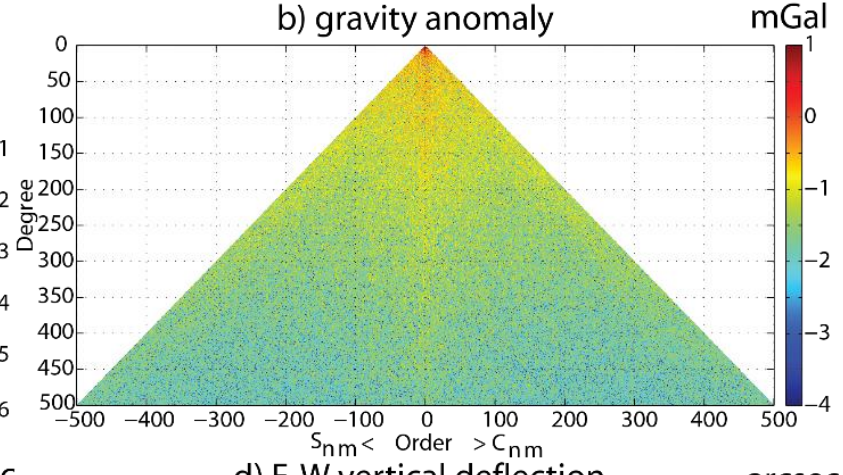

d) E-W vertical deflection

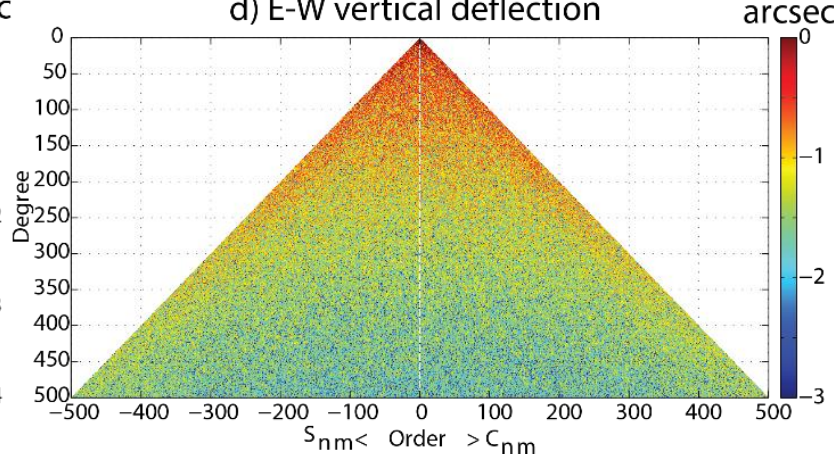

Figure 3. The results of the sensitivity analysis using EIGEN-6C model to degree and order 500 for a) geoid undulations in $\mathrm{m}, \mathrm{b}$ ) gravity anomaly in $\mathrm{mGal}, \mathrm{c}$ ) north-south vertical deflection in arcsec and d) east-west vertical deflection in arcsec. The quantities are the maximum signal size of the functionals contributed from each harmonic coefficient in $\log _{10}$ scale

\section{CONCLUSIONS}

The representation of geodetic or geophysical quantities in spherical coordinates using a 2D Fourier series has been confined in the literature to a scalar field like geopotential. In this paper, the 2D Fourier series is derived by transformation of the spherical harmonics from the Earth-fixed frame to a rotated frame. As a direct consequence, the Fourier series representation on the sphere is generalized to any linear functional of the scalar field. This generalization is done by using spectral transfers that relate the gradient of the field to the field itself. Each spectral transfer describes the spectral properties of the corresponding gradient which can be used for different spectral and spatial analyses on the sphere. 
Three numerical examples were provided to show the applicability of the 2D Fourier series representation. They included global spherical harmonic synthesis of the third-order gradient tensor, global spherical harmonic analysis of a global relief model and sensitivity analysis of the geoid undulations, gravity anomaly, N-S and E-W deflections of the vertical.

It should be mentioned that although the global spherical harmonic analysis is applied to a geometrical and isotropic quantity like DEM data, one can employ the representation presented in this paper to any isotropic to anisotropic functional. The global spherical harmonic analysis of anisotropic functionals makes this formulation a viable alternative to the spherical harmonic representation.

\section{REFERENCES}

Amante, C., \& Eakins, B. W., 2009. ETOPO1 1 arc-minute global relief model: procedures, data sources and analysis. US Department of Commerce, National Oceanic and Atmospheric Administration, National Environmental Satellite, Data, and Information Service, National Geophysical Data Center, Marine Geology and Geophysics Division.

Colombo, O. L., 1981. Numerical methods for harmonic analysis on the sphere (No. DGS-310). Ohio State University, Columbus, Department of Geodetic Science.

Ghobadi-Far, K., Sharifi, M. A., Sneeuw, N., 2015. 2D Fourier series representation of gravitational functionals in spherical coordinates. Submitted to Journal of Geodesy.

Gruber, C., Novák, P., Sebera, J., 2011. FFT-based highperformance spherical harmonic transformation. Studia Geophysica et Geodaetica, 55(3), pp. 489-500.

Heiskanen, W., \& Moritz, H., 1967. Physical geodesy. WH Freemann, San Francisco.

Jekeli, C., 1996. Spherical harmonic analysis, aliasing, and filtering. Journal of Geodesy, 70(4), pp. 214-223.

Ricardi, L. J., \& Burrows, M. L., 1972. A recurrence technique for expanding a function in spherical harmonics. IEEE Transactions on computers, (6), pp. 583-585.

Schuster, A., 1903. On Some Definite Integrals, and a New Method of Reducing a Function of Spherical Co-Ordinates to a Series of Spherical Harmonics. Philosophical Transactions of the Royal Society of London. Series A, Containing Papers of a Mathematical or Physical Character, pp. 181-223.

Shako, R., Förste, C., Abrikosov, O., Bruinsma, S., Marty, J. C., Lemoine, J. M., Flechtner, F., Neumayer , H., Dahle, C., 2014. EIGEN-6C: A high-resolution global gravity combination model including GOCE data. In Observation of the System Earth from Space-CHAMP, GRACE, GOCE and future missions, pp. 155161

Sneeuw, N., 1992. Representation coefficients and their use in satellite geodesy. Manuscripta Geodaetica, (17), pp. 117-123.

Sneeuw, N., 2000. A semi-analytical approach to gravity field analysis from satellite observations. PhD thesis, University of Munich.
Sneeuw, N., \& Bun, R., 1996. Global spherical harmonic computation by two-dimensional Fourier methods. Journal of Geodesy, 70(4), pp. 224-232.

Šprlák, M., \& Novák, P., 2015) Integral formulas for computing a third-order gravitational tensor from volumetric mass density, disturbing gravitational potential, gravity anomaly and gravity disturbance. Journal of Geodesy, 89(2), pp. 141-157.

Wigner, E., 1959. Group theory: and its application to the quantum mechanics of atomic spectra. Academic, New York. 